Posters were also produced to disseminate information. In the subsequent NTS, there was an improvement in SABP's performance in 12/18 indicators in the Doctors in training survey, with one green flag denoting performance in the top quartile of trusts nationally.

Conclusion. The NTS can be analysed to create a plan of action with elements that trainers and trainees feel can improve their experience. Our model demonstrates the potential for using NTS data to plan quality improvement in training.

\section{Enhanced faculty development: using the GMC survey to run a trainer development workshop \\ Martin Schmidt ${ }^{1 \star}$ and Timothy Leung ${ }^{2}$ \\ ${ }^{1}$ Surrey and Borders Partnership NHS Foundation Trust and ${ }^{2}$ East London NHS Foundation Trust \\ ${ }^{*}$ Corresponding author.}

doi: 10.1192/bjo.2021.435

Aims. To investigate whether the General Medical Council (GMC) National Training Surveys (NTS) can be analysed to create a trainer development workshop that improves postgraduate training.

Background. As part of its role in quality assurance of medical training, the GMC conducts an annual survey of trainers and trainees. The Trainer survey, part of the NTS, consists of 47 questions which are grouped into 11 indicators of quality. At Surrey and Borders Partnership NHS Foundation Trust, we were keen to use the comprehensive data in the NTS to improve training. We analysed each question to create a workshop to engage trainers in discussion about improving the experiences of trainers and trainees.

Method. Our analysis of the NTS used data from the online reporting tool to calculate the scores that were obtained for each question in the 2018 NTS. A question was discussed at the workshop if it performed poorly relative to other questions in the indicator; to provide useful information; or to clarify ambiguity. Indicators where interesting comparisons can be drawn between the views of trainers and trainees were also discussed. The 90-minute workshop was led by the Leadership and Education Fellow and Director of Medical Education. Attendees were subsequently sent an online survey.

Result. The workshop consisted of an introduction to the NTS; group discussion on which indicators were felt to be important, good- or poor-performing; discussion of specific questions; and a review of feedback from trainees.

12 questions and 3 indicators (Handover, Supportive environment, Rota design) were discussed. 11 questions were chosen for poor performance, which sought to contextualise the results within the experience of attendees. 8 questions were chosen to provide information, such as resources and current initiatives. 3 were chosen to clarify ambiguity. Many questions met several criteria.

17 attendees responded to the online survey. $64.7 \%$ agreed or strongly agreed that the NTS asks questions that are important for them. $76.5 \%$ agreed or strongly agreed that the NTS can be used to improve the trainer experience.

In the subsequent NTS, there was an improvement in 9/11 indicators in the Trainer Survey, with four green flags denoting performance in the top quartile of trusts nationally.

Conclusion. The NTS can be used to structure a workshop that trainers feel can improve their experience. Our strategy demonstrates the value of analysing the NTS dataset intelligently to engage trainers in improving training.
Is it possible to use research to learn psychiatry from scratch: a reflective self-study of a pre-clinical year medical student

Jashan Selvakumar ${ }^{1 *}$, Jiann Lin Loo $^{2}$, May Honey $\mathrm{Ohn}^{3}$ and Gabby Kelly ${ }^{4}$

${ }^{1}$ St Georges University of London; ${ }^{2}$ Betsi Cadwaladr University Health Board; ${ }^{3}$ University Hospital Lewisham and ${ }^{4}$ Cardiff University ${ }^{\star}$ Corresponding author.

doi: 10.1192/bjo.2021.436

Aims. Despite the abundance of opportunities available for medical students to explore the field of psychiatry, active immersion through experiential learning has proven to be difficult for preclinical year students as a result of a busy time table and the need to wait for psychiatry postings during the clinical years. Hence, the question of "how to implement experiential learning of psychiatry in pre-clinical years" arises. This study is aimed to elucidate the attempts that have been made to use research as a proximate approach to learn psychiatry experientially, focusing specifically on the challenges faced and lessons learned by a preclinical medical student.

Method. This self-study outlined the informal three-months learning-by-doing journey of a year-one medical student, supervised by a psychiatrist registrar. Employing research as a proximate approach of experiential learning for psychiatry was explored based on reflection from discussion during supervision meetings and messages exchange. The agreed learning method was an active involvement in research projects on psychiatry topics, with the learning outcome of producing publications.

Result. The challenges faced included: 1) the difficulty associated with striking a balance between an ambitious project with high impact versus a feasible smaller project to keep both parties motivated through the means of short-term accomplishment; 2) the ongoing requirement for learning process adjustment to build the foundational knowledge essential for progress. Through active and deliberate effort, every step in the process was found to be an opportunity for active learning. Literature review, for example, was used to build the understanding of psychiatry topics and practise critical appraisal skills, while allowing for the recognition of knowledge gaps, which ultimately encouraged future research idea synthesis. The process of writing and submitting a manuscript was used to learn publication-relevant skills including: journal impact calculation, referencing, indexing and abstracting services, and publication ethics. Certain future proof skills were also developed, including literacy in information and communication technology which improved efficiency of research, problem solving and decision making. This was done using pros and cons whenever difficulties were faced.

Conclusion. Although research is not a comprehensive substitute for clinical posting in the process of learning psychiatry, the lessons learned from psychiatry research can potentially serve as an initial exploration tool for preclinical-year medical students interested in the field. The stimulating process has found to be effective in stimulating further interest in psychiatry but maintaining it will be the next challenge.

Improving the confidence and competence of junior doctors in conducting seclusion reviews

Mostafa Shalaby* and Mehtab Rahman

Central \& North West London NHS Foundation Trust ${ }^{\star}$ Corresponding author.

doi: 10.1192/bjo.2021.437 
Aims.

- To improve the quality and consistency of medical seclusion reviews at St Charles Hospital and across the Trust.

- To ensure at least $80 \%$ compliance with minimum standards for seclusion review documentation by the end of December 2020.

- To increase doctors' mean perceived competence and confidence scores to $4.5 / 5$ by the end of December 2020

Method. Seclusion is commonly used to manage patients at high risk of aggression or violence, but is a high risk and very restrictive intervention. As such, it requires regular nursing and medical reviews. Work has been done recently at St Charles to improve the timeliness and effectiveness of nursing reviews including detailed guidance. Medical reviews are usually performed by junior doctors, many with limited experience in psychiatry. There is

- A lack of consistent local or national guidance for junior doctors undertaking seclusion reviews

- The quality and scope of these reviews is not consistent

- There may be a need to ensure that there is more standardization and to improve junior doctors' confidence - and therefore patient safety and experience - overall.

- The following interventions were used to improve the quality of seclusion reviews at the hospital:

- Minimum standard guidelines

- Presenting in Restrictive interventions meeting.

- Feedback from PICU consultants for guidelines

- Changing guidelines

Future plans:

- Guidelines teaching (Early November)

- Re-audit and new survey (Early November)

- Simulation training (Mid November)

- Seclusion teaching video (Early December- to be ready for Induction)

- Re-audit and new survey (Beginning of April)

Result. Surveys were conducted before and after quality improvement interventions were put in place. The average confidence levels of junior doctors increased from $38.5 \%$ to $87 \%$ following these interventions.

Conclusion. Revision of seclusion guidelines, junior doctor teaching and simulation training are effective interventions to improve junior doctor confidence levels in conducting seclusion reviews.

\section{Impact of mental health and addiction NIMHANS} ECHO on primary care physicians: study from a rural state of India

Shabinabegam A M Sheth ${ }^{1 \star}$, Bhavya Bairy ${ }^{2}$, Aurobind Ganesh ${ }^{2}$, Sumi Jain ${ }^{3}$, Prabhat Chand ${ }^{2}$, Pratima Murthy ${ }^{2}$ and Sanjeev Arora ${ }^{4}$

${ }^{1}$ Central and North West London NHS Foundation Trust, Dept of Psychiatry, National Institute of Mental Health and Neurosciences;

${ }^{2}$ Dept of Psychiatry, National Institute of Mental Health and Neurosciences; ${ }^{3}$ State Health Society and ${ }^{4}$ Project ECHO, University of New Mexico Health Science Centre

${ }^{\star}$ Corresponding author.

doi: $10.1192 /$ bjo.2021.438

Aims. As per National Mental Health Survey-2015-16, 83 out of 100 people having mental health problems do not have access to care in India. Further, primary health care providers (PCPs) have not been adequately trained in the screening, diagnosis, and initial management of common mental health conditions. There is thus a need to train health care providers at the State level to incorporate mental health into primary health care. In this paper, we report the findings of a collaborative project between the National Institute of Mental Health and Neuro Sciences (NIMHANS) Bangalore India, and the state of Chhattisgarh incorporating mental health into primary care and addressing urban-rural disparities through tele-mentoring.

Method. We assessed the impact of the NIMHANS Extended Community Health Care Outcome (ECHO), an online, blended training program on participants' knowledge and competence (primary outcome) and commitment, satisfaction, and performance (Secondary outcomes) using Moore's evaluation framework. Primary and secondary outcomes were determined through a prepost evaluation, assessment of trainee participation in the quarterly tele ECHO clinic as well as periodic assignments, respectively.

Result. Over ten months of the NIMHANS ECHO program, there was a significant improvement in the participants' knowledge post-ECHO $(\mathrm{p}<0.05, \mathrm{t}=-3.52)$. Self-efficacy in diagnosis and management of mental health problems approached significance; $p<0.001$. Increased engagement in tele-ECHO sessions was associated with better performance for declarative and procedural knowledge. The attrition rate was low (5 out of 30 dropped out), and satisfaction ratings of the course were high across all fields. The participants reported a 10- fold increase in the number of patients with mental health problems they had seen, following the training. A statistically significant increase in the number of psychotropic drugs prescribed post ECHO with $\mathrm{t}=-3.295, \mathrm{p}=0.01$.

Conclusion. The outcomes indicate that the NIMHANS ECHO with high participant commitment is a model with capacity building potential in mental health and addiction for remote and rural areas by leveraging technology. This model has the potential to be expanded to other states in the country in providing mental health care to persons in need of care.

Core Trainees' study budget and study leave: a survey exploring trainees' needs and understanding of local processes in place within the North West

\section{Chirag Shroff \\ CAMHS, Burlington House, Alder Hey NHS Foundation Trust}

\section{doi: 10.1192/bjo.2021.439}

Aims. Health Education England launched a new system for study leave and study budget on 1st April 2018, in response to trainees' concerns regarding the previous system. According to this, Health Education England would manage the study leave budget through its local offices, making the process of accessing study 'more transparent, equitable and streamlined' for all trainees. At the RAP Oversight Committee meeting of the North West Deanery in 2019, trainees' uncertainties over the process was discussed by the local reps. It was aimed that there was a need to gather information on trainees' needs and understanding of local processes in place by the deanery to access study leave and study budget

Method. A cross sectional survey was sent out to all the trainees by the Core Trainees year 1 RAP rep. A total of 6 relevant questions were designed and sent out to the trainees, allowing them 2 weeks' time to respond. There were a total of 66 trainees who were sent the survey. The guidance mentioned in the 2016 Gold guide was used for reference to ensure the questions are relevant.

Result. Of the total of 66 trainees who were sent the survey, there were 48 respondents. The results indicated that all 48 responders 